\title{
Prenatal exposure to paracetamol/acetaminophen and precursor aniline impairs masculinisation of male brain and behaviour
}

\author{
Anders Hay-Schmidt ${ }^{1}$, Olivia T Ejlstrup Finkielman ${ }^{1}$, Benjamin A H Jensen², \\ Christine F Høgsbro ${ }^{2}$, Jacob Bak Holm², Kristoffer Haurum Johansen ${ }^{2}$, Tina Kold Jensen ${ }^{3}$, \\ Anderson Martino Andrade ${ }^{4}$, Shanna H Swan ${ }^{5}$, Carl-Gustaf Bornehag, ${ }^{5,6}$, Søren Brunak ${ }^{8}$, \\ Bernard Jegou ${ }^{7,9}$, Karsten Kristiansen² and David Møbjerg Kristensen ${ }^{7,8}$ \\ ${ }^{1}$ Department of Neuroscience and Pharmacology, Faculty of Health and Medical Sciences, \\ University of Copenhagen, Copenhagen, Denmark, ${ }^{2}$ Department of Biology, Faculty of Science, University of \\ Copenhagen, Copenhagen, Denmark, ${ }^{3}$ Department of Growth and Reproduction, Rigshospitalet, University of \\ Copenhagen, Copenhagen, Denmark, ${ }^{4}$ Department of Physiology, Universidade Federal do Paraná, Curitiba, Brazil, \\ ${ }^{5}$ Department of Environmental Medicine and Public Health, Icahn School of Medicine at Mount Sinai, New York \\ City, New York, USA, ${ }^{6}$ Department of Health Sciences, Karlstad University, Karlstad, Sweden, ${ }^{7}$ Inserm (Institut \\ national de la santé et de la recherche médicale), IRSET, U1085, Rennes, France, ${ }^{8}$ Novo Nordisk Foundation Center \\ for Protein Research, University of Copenhagen, Copenhagen, Denmark, and ${ }^{9}$ EHESP-School of Public Health,
} Rennes, France

Correspondence should be addressed to D M Kristensen; Email: david.kristensen@cpr.ku.dk

\begin{abstract}
Paracetamol/acetaminophen (N-Acetyl-p-Aminophenol; APAP) is the preferred analgesic for pain relief and fever during pregnancy. It has therefore caused concern that several studies have reported that prenatal exposure to APAP results in developmental alterations in both the reproductive tract and the brain. Genitals and nervous system of male mammals are actively masculinised during foetal development and early postnatal life by the combined actions of prostaglandins and androgens, resulting in the male-typical reproductive behaviour seen in adulthood. Both androgens and prostaglandins are known to be inhibited by APAP. Through intrauterine exposure experiments in $\mathrm{C} 57 \mathrm{BL} / 6$ mice, we found that exposure to APAP decreased neuronal number in the sexually dimorphic nucleus (SDN) of the preoptic area (POA) in the anterior hypothalamus of male adult offspring. Likewise, exposure to the environmental pollutant and precursor of APAP, aniline, resulted in a similar reduction. Decrease in neuronal number in the SDNPOA is associated with reductions in male sexual behaviour. Consistent with the changes, male mice exposed in uteri to APAP exhibited changes in urinary marking behaviour as adults and had a less aggressive territorial display towards intruders of the same gender. Additionally, exposed males had reduced intromissions and ejaculations during mating with females in oestrus. Together, these data suggest that prenatal exposure to APAP may impair male sexual behaviour in adulthood by disrupting the sexual neurobehavioral programming. These findings add to the growing body of evidence suggesting the need to limit the widespread exposure and use of APAP by pregnant women.

Reproduction (2017) 154 145-152
\end{abstract}

\section{Introduction}

The foetal brain is bipotential in its ability to adopt a masculine or feminine phenotype; a property lost after a foetal and perinatal sensitive period (McCarthy 2008). The foetal male brain requires gonadal hormones, such as testosterone, to differentiate into the male configuration resulting in male reproductive behaviour later in life (Juntti et al. 2010). It is known that testosterone in the hypothalamus through its conversion to estradiol increases prostaglandin E2 (PGE2) by upregulating cyclooxygenase-2 (COX-2) resulting in masculinisation (Amateau \& McCarthy 2004). Accordingly, perinatal exposure to the COX enzyme inhibitor, acetylsalicylic acid (aspirin), results in the impairment of male sexual behaviour (Amateau \& McCarthy 2004). It is also known that intrauterine exposure to phthalates (like dibutyl phthalate), which also are inhibitors of COX enzymes with potency in the same range as mild analgesics (Kristensen et al. 2011a,b), can result in feminised play behaviour among young boys (Swan et al. 2010). At the same time, it is becoming increasingly clear that 
mild analgesics (hereafter termed analgesics) are potent inhibitors of foetal androgen production and through this mechanism can result in congenital malformations (reviewed in Kristensen et al. 2016). Hence, it seems possible that analgesics can affect brain masculinisation both by blocking the production of testosterone in testicular Leydig cells and prostaglandins in the brain. Moreover, previous studies have shown that postnatal exposure (on postnatal day 10) to paracetamol/ acetaminophen (N-Acetyl-p-Aminophenol; APAP) can induce long-lasting effects on e.g. cognitive function in the adult mouse (Viberg et al. 2014).

In the present report, we therefore examined mouse brain morphology and behaviour following prenatal exposure to APAP, used widely among pregnant women (Kristensen et al. 2016), and the industrial pollutant and APAP precursor, aniline (Modick et al. 2014, Holm et al. 2015). We investigated changes in the sexually dimorphic nucleus (SDN) of the preoptic area (POA) in the anterior hypothalamus of male adult offspring after intrauterine exposure. The SDN has been shown to be 2.5-7 times larger in male than that in female rodents, with the difference being dependent on induced levels of PGE2 resulting from the action of testosterone (Amateau \& McCarthy 2004). Similar sex differences in size of this nucleus have been described in other mammals (Morris et al. 2004, Gilmore et al. 2012). The calcium-binding protein calbindin-D28 is one of the best markers for identifying the anatomical borders of the SDN-POA in mice (Sickel \& McCarthy 2000, Gilmore et al. 2012). The protein belongs to a family of low-molecular-weight calcium-binding proteins that has been hypothesised to play a role in neuroprotection against excitatory neurotransmitter and calcium-induced cell death (Sickel \& McCarthy 2000). In rodents, neurogenesis of SDN neurons begins around embryonic day 14 and proceeds until about embryonic day 18 , and all the neurons in the surrounding area of the POA have been created by embryonic day 16 in the rodent (Jacobson \& Gorski 1981). Our findings indicate a potential effect of APAP on intrauterine brain development and suggest that these changes may have a direct effect on male sexual behaviour.

\section{Materials and methods}

\section{Mating and dosing}

The local Danish ethical committees approved all animal experiments. Mice (Mus musculus) dams with a C57BL/6)BomTac background ( $n=10$ per group, purchased from Taconic, Denmark) were fed on a phytoestrogen-free diet. The dams were randomly caged pairwise with a male during mating. Pregnant dams were subsequently treated every morning at the end of the dark period from 7 days post coitum (DPC) to delivery following dosing protocols also described in the study by Holm and coworkers $(2015,2016)$. Compounds were administered in $0.5 \mathrm{~mL}$ water by gavage in the following groups: (i) water control, (ii) $50 \mathrm{mg} / \mathrm{kg} /$ day APAP, (iii) $150 \mathrm{mg} /$ $\mathrm{kg} /$ day APAP, (iv) $30 \mathrm{mg} / \mathrm{kg} /$ day aniline and (v) $90 \mathrm{mg} / \mathrm{kg} /$ day aniline. APAP doses were chosen to be nontoxic and the lower to be equal to the maximum human exposure of $50 \mathrm{mg} / \mathrm{kg} /$ day. The higher dose of $150 \mathrm{mg} / \mathrm{kg} /$ day was chosen so it also was in the range of the human exposure when a system of allometry based on the body surface area difference between mouse and human was applied dividing the mice dose with a factor of 12.33 (Reagan-Shaw et al. 2008). Aniline doses were given in equi-molar amounts to the APAP doses and were, after adjusting for difference in body size between mouse and human, also within the human exposure range (Holm et al. 2015, 2016).

\section{Analysis of the sexually dimorphic nucleus of the preoptic area}

After termination at PNW 12-13, brains were carefully dissected from the skull and examined to explore any changes in SDN-POA. Brains were fixed in PBS (phosphate buffered saline) with $4 \%$ paraformaldehyde and subsequently split in half in the coronal plane (where the midbrain and the thalamus meet) and submerged in $30 \%$ sucrose to avoid freezing damage. From each of the treatment groups brains were chosen at random. Coronal sections of each brain were cut into four series at $40 \mu \mathrm{m}$ on a sledge microtome. Series were stored at $-20^{\circ} \mathrm{C}$ in cryoprotectant with sodium azide ( $\mathrm{NaAZ}$ ).

Before immunohistochemical staining, free-floating sections were rinsed three times for $10 \mathrm{~min}$ in PBS and subsequently incubated for $15 \mathrm{~min}$ in $0.5 \%$ perhydrol $\left(\mathrm{H}_{2} \mathrm{O}_{2}\right)$ to block endogenous peroxidase that could otherwise lead to falsepositive results in the non-specific background. Sections were rinsed in PBS again before being pre-incubated with human serum albumin (HSA) in PBS-T (1\% HSA, PBS-T, 0.1\% NaAZ) for $30 \mathrm{~min}$ to block unspecific absorbance to tissue or to $\mathrm{FC}$ receptors and permeabilize the tissue. After this, the sections were exposed to primary rabbit anti-Calbindin-D-28K (1:2000; Sigma-Aldrich; EG-20) antibody overnight in 1\% HSA-PBSTNaAZ. Next morning, the sections were rinsed in PBS-T and incubated with secondary donkey anti-rabbit antibody for $1 \mathrm{~h}$ in $1 \%$ HSA-PBST-NaAZ. This was followed by another rinse in PBS and incubation with an avidin-biotin (AB) complex (1:100; Vector-labs) in PBS for $1 \mathrm{~h}$. The sections were then rinsed in PBS and incubated in Vector SG-peroxidase HRP substrate kit (Vector-labs) for 15 min for development. Finally, the sections were rinsed twice in MilliQ ultrapure water and mounted in chrome-gelatine onto slides.

\section{Defining the area of the SDN-POA}

The procedure described by Gilmore and coworkers (2012) was used to distinguish the calbindin D28 immunoreactive part of the SDN (CALB-SDN) from other calbindin immunoreactive (CALB-ir) cells found in the C57BL/6 mouse brain. The CALB$\mathrm{SDN}$ is defined as a distinctive cluster that is ellipsoidal in shape and centred at about $675 \mu \mathrm{m}$ dorsal to the optic chiasm (OC) and $275 \mu \mathrm{m}$ lateral to the third ventricle. It is angled away from the third ventricle dorsolaterally and the cluster spans between $90 \mu \mathrm{m}$ and $180 \mu \mathrm{m}$ rostrocaudally in males (Gilmore et al. 2012). 
Depending on the exact plane of sectioning, the cluster was present in sections in which the anterior commissure (AC) crossed the midline or, more often, just caudal to this point. Using these landmarks to pinpoint the correct location of CALB-SDN, sections were chosen with the aid of the mouse brain atlas of Paxinos and Franklin (2001). Especially in specimens where the calbindin-reactive cells did not form a distinct cluster or form a very small cluster (Gilmore et al. 2012), the presence of these landmarks was the determining factor in the identification of the correct location.

\section{Quantifying the CALB-SDN}

The tissue sections were examined using an Evolution MP Color camera from Media Cybernetics connected to a light microscope and analysed with the aid of Image-Pro Plus and ImageJ software. All measurements were made on images coded to conceal the sex and treatment of the animals. For every brain in the experiment, one section was chosen that best represented the CALB-SDN. The correct location was determined as described in the above section, and if the CALB-SDN was visible in multiple sections, the section was chosen that had the most CALB-ir cells in the CALB-SDN. For the images in the group where a CALB-SDN was discernible, an ellipse of a fixed size was placed on both sides of the third ventricle in approximately the same location in all images relative to two markers, the third ventricle and the OC. The placement differed from what is described by Gilmore and coworkers (2012) in that it was consistently closer to the OC, approximately $400 \mu \mathrm{m}$ instead of $675 \mu \mathrm{m}$. The cells within the elliptical border were subsequently counted independently by two investigators. Importantly, if a CALB-ir cell was lying at the edge of the ellipse and was too far from the cluster of the other cells, it was counted as a background staining and not as part of the CALB-SDN. The number of cells from counts on both sides of the third ventricle was noted and added together for a representative CALB-SDN cell number from that brain.

\section{Behavioural testing}

From postnatal week eight (PNW 8), one male was selected at random from each of 8 litters that were prenatally exposed to $150 \mathrm{mg} / \mathrm{kg} /$ day APAP during intrauterine life and compared to an equal number of similarly chosen controls to explore (i) urinary behaviour, (ii) aggression and (iii) sexual behaviour. All behavioural assays were conducted $1 \mathrm{~h}$ after initiation of the dark cycle and the protocols used were adapted from Juntti and coworkers (2010) and Yang and coworkers (2013) with males single caged and with $2 \geq$ days separating each of the experiments. The tests were videotaped and subsequently analysed blindly by two investigators.

The urinary behaviour in males and females differ by the size of the urine spots, numbers and spread. Hence, a territorymarking male mouse will be more prone to marking their territory with urine scent and leave multiple, widespread spots, while a female will more commonly choose one or few areas where she will leave fewer, bigger droplets (Juntti et al. 2010). Males were tested by transferring the animal for $1 \mathrm{~h}$ to a cage freshly lined with Whatman paper and then returned to their home cage. The marking pattern was visualized with UV transillumination and the imaging program Image $(\mathrm{NIH})$.

Intermale aggression was provoked by the introduction of an intruder male (Barkley \& Goldman 1977). A singly-housed intruder mouse was introduced to the home cage of a singlyhoused resident mouse (prenatally treated). The intruder test was conducted for $15 \mathrm{~min}$ and sniffs, attacks, tail rattles and bites of the resident mouse were counted.

Male mating/sexual behaviour was tested for $30 \mathrm{~min}$ with a receptive intruder female in oestrous. During mating, the male mouse behaviour was documented by quantifying approach and olfactory investigation of the female (anogenital sniffing), mounting, intromission (penetration identified as thrusting) and ejaculation (Swaney et al. 2012).

\section{Statistics}

A one-way ANOVA follow by post hoc Dunnett's tests were used to determine the significance between outcomes in the neuronal examinations. Unpaired $t$-tests were performed between outcomes in behavioural trials.

\section{Results}

\section{Intrauterine exposure to APAP and aniline decreased cell number in the CALB-SDN}

There was no difference between animals at the lowest dose of APAP and control; mean numbers of CALB-ir cells were 44 and 41 in control and APAP-treated animals respectively. In contrast, exposure to the highest dose $(150 \mathrm{mg} / \mathrm{kg} /$ day $)$ significantly decreased cell number in the SDN by approximately $50 \%$ with a mean cell number of 21 (Fig. 1). The decrease seen for anilineexposed males was similar to that seen for the highest dose of APAP. Hence, both aniline doses resulted in a decrease in CALB-ir cell number in the CALB-SDN with significant decrease for the highest dose resulting in a mean cell number of 21 . These data suggest that the intrauterine exposure may have impaired the brain masculinisation process.

\section{Intrauterine exposure to APAP changed urinary marking behaviour}

Aniline is known to be converted nearly completely in the liver of the mouse to APAP (Holm et al. 2015). We therefore performed behavioural experiments with animals exposed to the highest dose of APAP (150 mg/ $\mathrm{kg} /$ day), which had resulted in a significant effect on masculinisation of the SDN-POA. The area covered by urine markings in the cages did not differ between control and APAP-exposed animals, as both covered approximately $13 \%$ of the cage with urinary marks (Fig. 2). In contrast, APAP-exposed animals had marked the cages with significantly larger droplets. Quantifying the number of droplets from each animals using a 'cut-off' of $>2000$ pixels per droplet showed that the intrauterine 

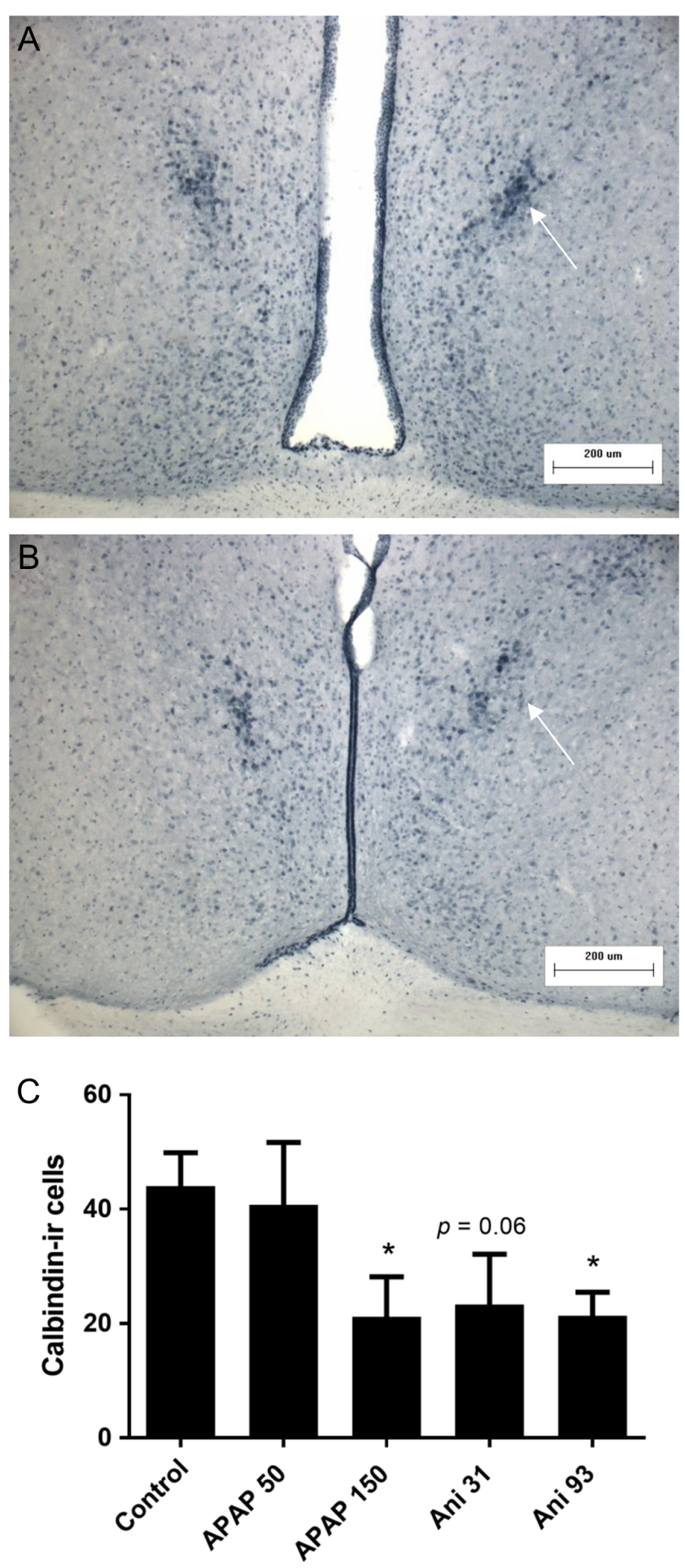

Figure 1 Prenatal exposure to paracetamol (APAP) and aniline from 7 days post coitum to delivery decrease neuronal number in the sexually dimorphic nucleus (SDN) of the preoptic area (POA) in the anterior hypothalamus of male adult offspring. (A and B) Decrease in the size of the SDN-POA (shown by arrows on the right side of the third ventricle) detected by staining for calbindin D28 (calbindin-ir) in adult male mouse. The study found no difference in SDN-POA size between controls and animals exposed to $50 \mathrm{mg} / \mathrm{kg} /$ day APAP. exposed males had marked their cages with significantly fewer but bigger droplets (Fig. 2B and C). These data suggest that the APAP exposure may have resulted in a less masculinised territorial marking of the cages.

\section{Intrauterine exposure to APAP resulted in reduced territorial display}

The male mouse is characterized by fierce aggression towards foreign males. We therefore introduced male intruders into the cages of the intrauterine exposed animals. Following the males interaction for $30 \mathrm{~min}$, we observed a significantly reduced number of sniffs among exposed animals $(150 \mathrm{mg} / \mathrm{kg} /$ day $)$ and a similar trend in tail rattles (Fig. $3 \mathrm{~A}$ and $\mathrm{C}$ ). While the number of direct attacks did not differ between exposed and control animals, the number of attacks that resulted in bites was markedly different; none of the APAP-exposed males bit their intruder (Fig. 3B and D). These data suggest that the intrauterine exposure to APAP may have moderated male-typical aggression during territorial display.

\section{Intrauterine exposure to APAP resulted in reduced mating}

The male mouse begins sexual behaviour immediately when introduced to a female in oestrus. As the urine marking and aggressiveness towards an intruding male had indicated that the male specific behaviour could be reduced in the APAP $(150 \mathrm{mg} / \mathrm{kg} /$ day $)$ exposed males, we next introduced a female in oestrus to males for 30 min to investigate the mating behaviour. Examining the behaviour, there were no changes between APAP and control mice in sniffing or mounting (Fig. 4A and B). However, moving from mounting to intromission, there was a clear decline in APAP mice (Fig. 4C). This was further supported when examining the females for vaginal plugs. $50 \%$ of the females encountering a control mouse had vaginal plugs after the 30-min experiment lasted, while none of the females introduced to an APAP male had plugs (Fig. 4D). These data suggest that the intrauterine exposure to APAP may have reduced the final stages of male sexual behaviour.

\section{Discussion}

Recent years have seen several rodent and human studies reporting connections between intrauterine exposure to

Micrographs therefore here show the difference between exposure for (A) 50 and (B) $150 \mathrm{mg} / \mathrm{kg} /$ day APAP during prenatal life. (C) Decrease in the size of the SDN-POA detected by staining for calbindin D28 (calbindin-ir) in adult male mouse after exposure to vehicle/water $(n=7), 50 \mathrm{mg} / \mathrm{kg} /$ day APAP $(n=6), 150 \mathrm{mg} / \mathrm{kg} /$ day APAP $(n=7), 31 \mathrm{mg} / \mathrm{kg} /$ day aniline $(n=6)$ and $93 \mathrm{mg} / \mathrm{kg} /$ day aniline $(n=9)$. Results are depicted as mean \pm S.E.M. and evaluated with a one-way ANOVA follow by post hoc Dunnett's test; ${ }^{*} P<0.05$. 

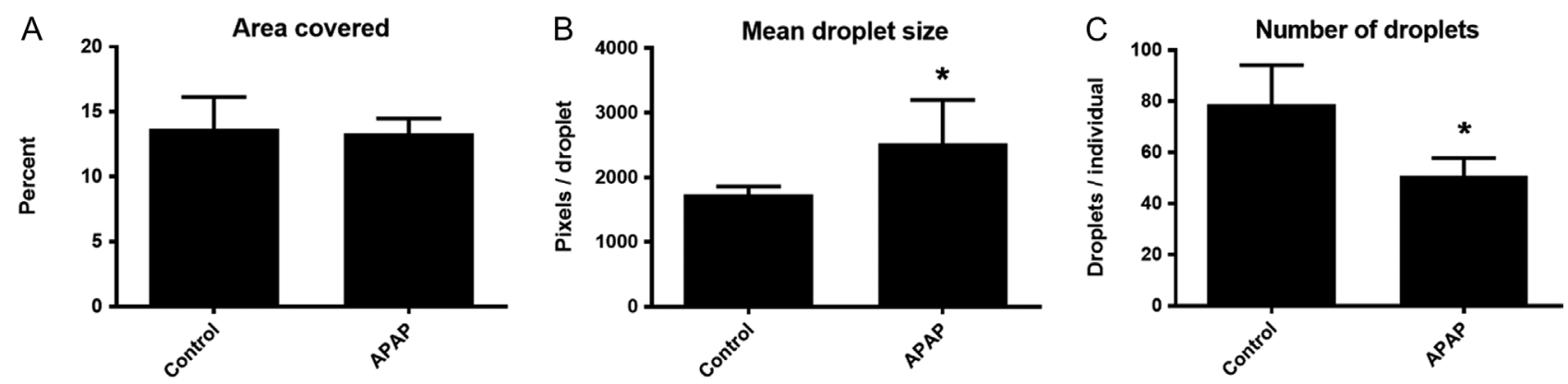

Figure 2 Intrauterine exposure to paracetamol $150 \mathrm{mg} / \mathrm{kg} /$ day (APAP) changes male territorial urine marking. (A) No change was found between APAP males and controls in area covered by urine marking. (B) APAP males deposited fewer urine marks compared to controls. (C) APAP males deposit larger urine marks compared to controls. Results are depicted as mean \pm S.E.M. and evaluated with an unpaired $t$-test; $n=8 ;{ }^{*} P<0.05$.

APAP and male congenital reproductive malformations and other morphological changes. Accordingly, APAP has been found to increase the risk of cryptorchidism and reduced anogenital distance through its anti-androgenic effects; both associated with later life reproductive disorders (reviewed in Kristensen et al. 2016).

With this exploratory study, we now report that the anti-androgenic effect of APAP on reproduction may not be restricted to urogenital development but could also include an effect on the masculinisation processes of the brain. APAP is clinically the preferred compound to treat pain and fever during pregnancy (Kristensen et al. 2016). The actions of APAP remain partly elusive, but one mechanism is through its inhibition of prostaglandin synthesis (Kristensen et al. 2011a,b). Prostaglandins are oxylipins that induce both pain and fever, while also being intrinsically involved in the brain masculinisation process during foetal life by translating the testosterone signal originating from testis to the SDN-POA (Amateau \& McCarthy 2004). The convergence of both prostaglandin and testosterone inhibitory effects are likely the reason for the reduction in CALB-ir cell number in the SDN-POA. However, in the present report, we cannot distinguish between the effect on testosterone and prostaglandin, as evidence also suggests a direct role for androgens in the brain masculinisation process. Thus, androgen receptor (AR)-deficient mice exhibit results in reduced CALBSDN cell number and an intermediate phenotype not significantly different from females (Edelmann et al. 2007).

In our behaviour experiments, the pattern of urine marking appeared to be independent of the intrauterine APAP exposure as the exposed males distributed their urine marks across the cage floor similarly to control males. However, the exposed males did have fewer and larger urine marks compared to control males. Interestingly, a previous report, using a brain-specific
A

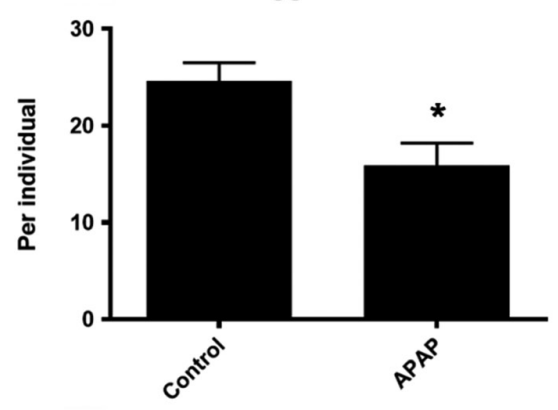

C

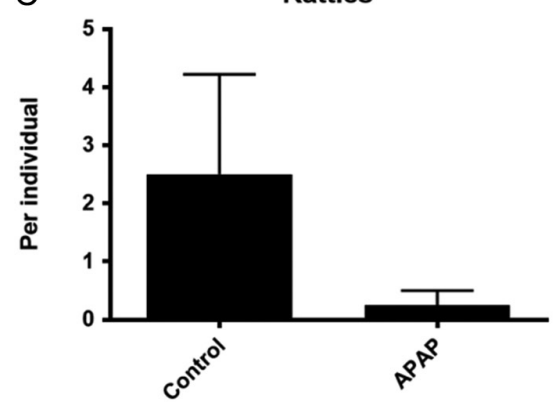

B

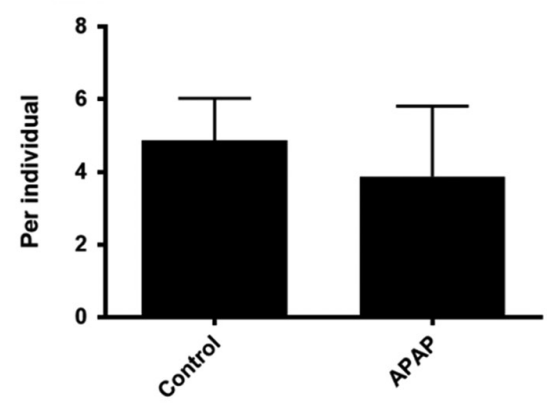

D

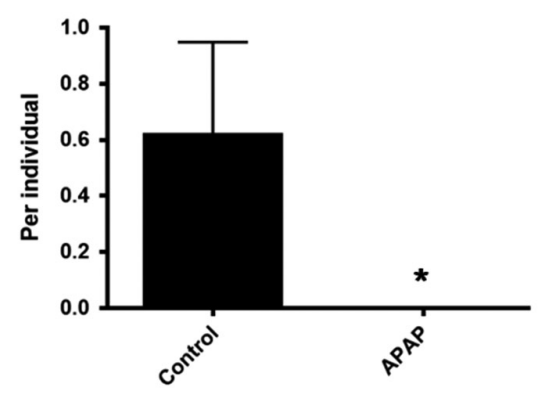

Figure 3 Intrauterine exposure to $150 \mathrm{mg} / \mathrm{kg} /$ day paracetamol (APAP) moderates male territorial display and fighting. (A) APAP males have fewer aggressive sniffs compared to controls. (B) No change was found between APAP males and controls in number of attacks. (C) APAP males have a tendency to less tail rattles compared to controls. (D) APAP males did not bite intruding males in contrast to controls. Results are depicted as mean \pm S.E.M. and evaluated with an unpaired $t$-test; $n=8$; $* P<0.05$. 
A

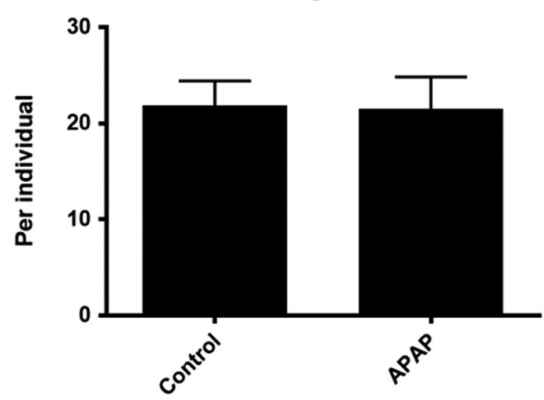

C

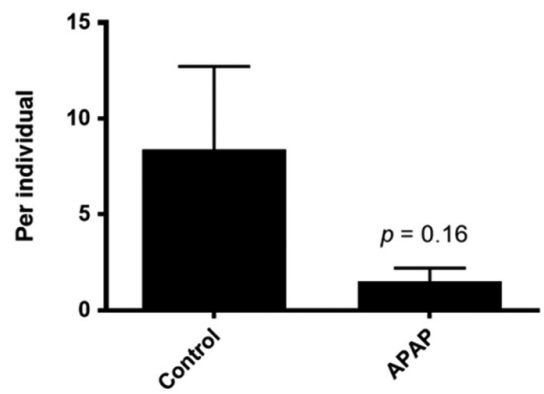

B

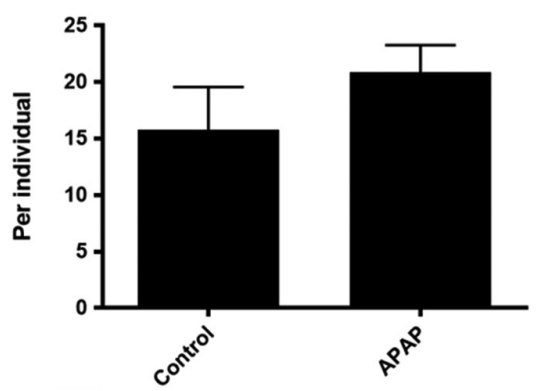

D

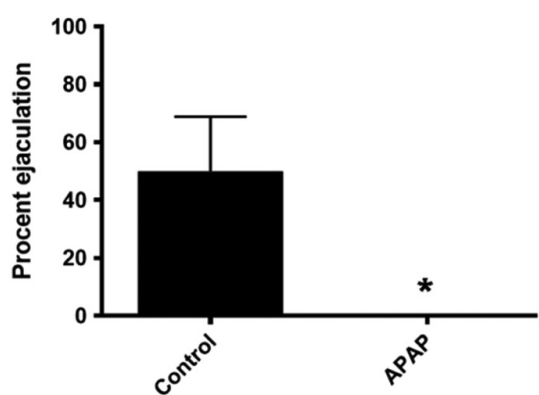

Figure 4 Intrauterine exposure to $150 \mathrm{mg} / \mathrm{kg} /$ day paracetamol (APAP) decreased male mating behaviour. (A) No change was found between APAP males and controls in mating sniffs. (B) No change was found between APAP males and controls in mounting females. (C) APAP males had a tendency to fewer intromissions with females in oestrus compared to controls. (D) APAP males did not ejaculate during intromission in contrast to controls. Results are depicted as mean \pm S.E.M. and evaluated with an unpaired $t$-test; $n=8$; $* P<0.05$. deletion of AR in mice, has shown that androgens are not required to masculinise urine marking (Juntti et al. 2010). Rather, AR enhances the male display of urine marking, increasing the number of urine marks and the male pattern. With these data in mind, the anti-androgenic effect of APAP could account for the fewer and larger markings among the intrauterine exposed males.

Both male mice with lesions in the POA and rats with decreased SDN-POA cell number have impaired mounting behaviour and copulation (Houtsmuller et al. 1994, Morris et al. 2004, Lin et al. 2011, Swaney et al. 2012). Furthermore, SDN-POA cell number has been reported to be significantly reduced in prenatally stressed rat males that did not copulate, and random control males with similar lack of copulation had similar reductions in cell number (Rhees et al. 1999). Moreover, specific lesions in the SDN-POA results in similar reductions in copulatory behaviour (De Jonge et al. 1989, Morris et al. 2004). It is therefore not surprising that the reduction in CALB-ir cell number in the SDNPOA reported here is correlated with a reduction in male sexual behaviour and lack of ejaculation. These data are also in accordance with a former rat study that showed that intrauterine and perinatal exposure to acetylsalicylic acid, an analgesic not recommended during pregnancy (Kristensen et al. 2016), resulted in reduced copulatory behaviour (Amateau \& McCarthy 2004).

As several dimorphic regions have been described in the brain, a limitation of the present report is that we cannot argue that the differences found in aggression and sexual behaviour are exclusively due to changes in the SDN-POA. Even the POA is known to harbour another site of sexual dimorphism termed the anteroventral periventricular (AVPV) nucleus where increased cell number is associated with feminine behaviour (Simerly 1989). The regions associated with mating and aggression do, however, exhibit spatial overlap in the brain (Lin et al. 2011). In some instances, these areas respond during both mating and aggression (Lin et al. 2011), showing that the behavioural schemes are both morphologically and functionally linked. Another limitation of the study is that the activities of these regions are likely also dependent on gonadal-pituitary axis e.g. the testosterone-LH ratio in adulthood, which was not investigated.

We included aniline in our experiments to explore both the pharmacological and the environmental exposure to APAP. Hence, it is becoming increasingly clear that APAP is presenting itself as a compound with a dual exposure pattern, including high doses through pharmaceutical use and lower doses through the environment possibly through the conversion of ubiquitous pollutant aniline and its derivatives (Kristensen et al. 2016). Exposure to aniline resulted in a similar reduction in CALB-ir cell number of the SDNPOA as APAP. The reason for this is likely that aniline is metabolized to APAP in the liver of the pregnant dam (Holm et al. 2015). We used an aniline exposure level corresponding to the amount of APAP allowed to be used by pregnant women $(50 \mathrm{mg} / \mathrm{kg} /$ day $)$ together with 3 times this amount, reaching a level known from rodent studies to inflict anti-androgenic effects (Kristensen et al. $2011 a, b)$. The lowest level of aniline exposure was $31 \mathrm{mg} / \mathrm{kg} /$ day, which we have previously shown to result in mean urinary concentrations of $309.9 \mathrm{mg} / \mathrm{L}$ APAP after $4 \mathrm{~h}$ in C57Bl/6 (Holm et al. 2015). In humans 
occupationally exposed to aniline, the range of APAP found in the urine is $4.2-10.9 \mathrm{mg} / \mathrm{L}$ (Dierkes et al. 2014). The exposure is therefore in proximity to the exposure observed among occupationally exposed humans.

In common practice, a system of allometry based on body surface area is used to translate animal to human doses. In this system, the mouse dose is divided by a factor of 12.33 to reach appropriate human levels (ReaganShaw et al. 2008). According to this estimate, the doses in this report resulting in significant effects of $150 \mathrm{mg} / \mathrm{kg} /$ day are well below the APAP doses used by pregnant women across Europe and the North America of $50 \mathrm{mg} / \mathrm{kg} /$ day. Consequently, these exploratory experiments have relevance to human health and follow previous reports suggesting an impact of APAP on brain development resulting in e.g. hyperkinetic disorders (Brandlistuen et al. 2013, Liew et al. 2014, Hoover et al. 2015).

\section{Declaration of interest}

No funding bodies or other agencies have had any role in study design, data collection and analysis, decision to publish, or preparation of the manuscript. There is no conflict of interest that could be perceived as prejudicing the impartiality of the research reported.

\section{Funding}

Funding for carrying out this study was kindly provided by The Danish Council for Independent Research (Medical Sciences).

\section{Acknowledgements}

Dorthe Meinertz and Dennis Madsen are gratefully acknowledged for support and assistance during the studies.

\section{References}

Amateau SK \& McCarthy MM 2004 Induction of PGE2 by estradiol mediates developmental masculinization of sex behavior. Nature Neuroscience 7 643-650. (doi:10.1038/nn1254)

Barkley MS \& Goldman BD 1977 The effects of castration and silastic implants of testosterone on intermale aggression in the mouse. Hormones and Behavior 9 32-48. (doi:10.1016/0018-506X(77)90048-4)

Brandlistuen RE, Ystrom E, Nulman I, Koren G \& Nordeng H 2013 Prenatal paracetamol exposure and child neurodevelopment: a sibling-controlled cohort study. International Journal of Epidemiology 42 1702-1713. (doi:10.1093/ije/dyt183)

De Jonge FH, Louwerse AL, Ooms MP, Evers P, Endert E \& van de Poll NE 1989 Lesions of the SDN-POA inhibit sexual behavior of male Wistar rats. Brain Research Bulletin 23 483-492. (doi:10.1016/03619230(89)90194-9)

Dierkes G, Weiss T, Modick H, Käfferlein HU, Brüning T \& Koch HM 2014 $\mathrm{N}$-Acetyl-4-aminophenol (paracetamol), N-acetyl-2-aminophenol and acetanilide in urine samples from the general population, individuals exposed to aniline and paracetamol users. International Journal of Hygiene and Environmental Health 217 592-599. (doi:10.1016/j.ijheh.2013.11.005)

Edelmann M, Wolfe C, Scordalakes EM, Rissman EF \& Tobet S 2007 Neuronal nitric oxide synthase and calbindin delineate sex differences in the developing hypothalamus and preoptic area. Developmental Neurobiology 67 1371-1381. (doi:10.1002/dneu.20507)
Gilmore RF, Varnum MM \& Forger NG 2012 Effects of blocking developmental cell death on sexually dimorphic calbindin cell groups in the preoptic area and bed nucleus of the stria terminalis. Biology of Sex Differences 3 5. (doi:10.1186/2042-6410-3-5)

Holm JB, Chalmey C, Modick H, Jensen LS, Dierkes G, Weiss T, Jensen BAH, Nørregård MM, Borkowski K, Styrishave B et al. 2015 Aniline is rapidly converted into paracetamol impairing male reproductive development. Toxicological Sciences 148 288-298. (doi:10.1093/toxsci/kfv179)

Holm JB, Mazaud-Guittot S, Danneskiold-Samsøe NB, Chalmey C, Jensen B, Nørregård MM, Hansen $\mathrm{CH}$, Styrishave B, Svingen T, Vinggaard AM et al. 2016 Intrauterine exposure to paracetamol and aniline impairs female reproductive development by reducing follicle reserves and fertility. Toxicological Sciences 150 178-189. (doi:10.1093/toxsci/kfv332)

Hoover RM, Hayes VAG \& Erramouspe J 2015 Association between prenatal acetaminophen exposure and future risk of attention deficit/ hyperactivity disorder in children. Annals of Pharmacotherapy 49 1357-1361. (doi:10.1177/1060028015606469)

Houtsmuller EJ, Brand T, de Jonge FH, Joosten RNJMA, van de Poll NE \& Slob AK 1994 SDN-POA volume, sexual behavior, and partner preference of male rats affected by perinatal treatment with ATD. Physiology and Behavior 56 535-541. (doi:10.1016/0031-9384(94)90298-4)

Jacobson CD \& Gorski RA 1981 Neurogenesis of the sexually dimorphic nucleus of the preoptic area in the rat. Journal of Comparative Neurology 196 519-529. (doi:10.1002/cne.901960313)

Juntti SA, Tollkuhn J, Wu MV, Fraser EJ, Tan S, Honda S, Harada N \& Nirao M 2010 The androgen receptor governs the execution, but not programming, of male sexual and territorial behaviors. Neuron $\mathbf{6 6}$ 260-272. (doi:10.1016/j.neuron.2010.03.024)

Kristensen DM, Hass U, Lesne L, Lottrup G, Jacobsen PR, Desdoitslethimonier C, Boberg J, Petersen JH, Toppari J, Jensen TK et al. 2011a Intrauterine exposure to mild analgesics is a risk factor for development of male reproductive disorders in human and rat. Human Reproduction 26 235-244. (doi:10.1093/humrep/deq323)

Kristensen DM, Skalkam ML, Audouze K, Lesné L, Desdoits-lethimonier C, Frederiksen H, Brunak S, Skakkebæk NE, Jégou B, Hansen JB et al. 2011 b Many putative endocrine disruptors inhibit prostaglandin synthesis. Environmental Health Perspectives 119 534-541. (doi:10.1289/ ehp.1002635)

Kristensen DM, Mazaud-Guittot S, Gaudriault P, Lesné L, Serrano T, Main KM \& Jégou B 2016 Analgesic use - prevalence, biomonitoring and endocrine and reproductive effects. Nature Reviews Endocrinology 13 381-393. (doi:10.1038/nrendo.2016.55)

Liew Z, Ritz B, Rebordosa C, Lee P-CC \& Olsen J 2014 Acetaminophen use during pregnancy, behavioral problems, and hyperkinetic disorders. JAMA Pediatrics 168 313-320. (doi:10.1001/jamapediatrics.2013.4914)

Lin D, Boyle MP, Dollar P, Lee H, Lein ES, Perona P \& Anderson DJ 2011 Functional identification of an aggression locus in the mouse hypothalamus. Nature 470 221-226. (doi:10.1038/nature09736)

McCarthy MM 2008 Estradiol and the developing brain. Physiological Reviews 88 91-124. (doi:10.1152/physrev.00010.2007)

Modick H, Weiss T, Dierkes G, Brüning T \& Koch HM 2014 Ubiquitous presence of paracetamol in human urine: Sources and implications. Reproduction 147 R105-R117. (doi:10.1530/rep-13-0527)

Morris JA, Jordan CL \& Breedlove SM 2004 Sexual differentiation of the vertebrate nervous system. Nature Neuroscience 7 1034-1039. (doi:10.1038/nn1325)

Paxinos G \& Franklin KBJ 2001 Mouse Brain in Stereotaxic Coordinates. Academic Press, San Diego, CA, USA; 2001.

Reagan-Shaw S, Nihal M \& Ahmad N 2008 Dose translation from animal to human studies revisited. FASEB Journal 22 659-661. (doi:10.1096/ fj.07-9574LSF)

Rhees RW, Al-Saleh HN, Kinghorn EW, Fleming DE \& Lephart ED 1999 Relationship between sexual behavior and sexually dimorphic structures in the anterior hypothalamus in control and prenatally stressed male rats. Brain Research Bulletin 50 193-199. (doi:10.1016/S03619230(99)00191-4)

Sickel MJ \& McCarthy MM 2000 Calbindin-d28k immunoreactivity is a marker for a subdivision of the sexually dimorphic nucleus of the preoptic area of the rat: Developmental profile and gonadal steroid modulation. Journal of Neuroendocrinology 12 397-402. (doi:10.1046/ j.1365-2826.2000.00474.x)

Simerly RB 1989 Hormonal control of the development and regulation of tyrosine hydroxylase expression within a sexually dimorphic population 
of dopaminergic cells in the hypothalamus. Brain Research: Molecular Brain Research 6 297-310. (doi:10.1016/0169-328X(89)90075-2)

Swan SH, Liu F, Hines M, Kruse RL, Wang C, Redmon JB, Sparks A \& Weiss B 2010 Prenatal phthalate exposure and reduced masculine play in boys. International Journal of Andrology 33 259-269. (doi:10.1111/ j.1365-2605.2009.01019.x)

Swaney WT, Dubose BN, Curley JP \& Champagne FA 2012 Sexual experience affects reproductive behavior and preoptic androgen receptors in male mice. Hormones and Behavior 61 472-478. (doi:10.1016/j.yhbeh.2012.01.001)

Viberg H, Eriksson P, Gordh T \& Fredriksson A 2014 Paracetamol (acetaminophen) administration during neonatal brain development affects cognitive function and alters its analgesic and anxiolytic response in adult male mice. Toxicological Sciences 138 139-147. (doi:10.1093/ toxsci/kft329)
Yang CF, Chiang MC, Gray DC, Prabhakaran M, Alvarado M, Juntti SA, Unger EK, Wells JA \& Shah NM 2013 Sexually dimorphic neurons in the ventromedial hypothalamus govern mating in both sexes and aggression in males. Cell 153 896-909. (doi:10.1016/j. cell.2013.04.017)

Received 20 March 2017

First decision 13 April 2017

Revised manuscript received 18 May 2017

Accepted 26 May 2017 